\title{
Trust in Anonymity Networks
}

\author{
Vladimiro Sassone, Sardaouna Hamadou, and Mu Yang
}

ECS, University of Southampton

\begin{abstract}
Anonymity is a security property of paramount importance, as we move steadily towards a wired, online community. Its import touches upon subjects as different as eGovernance, eBusiness and eLeisure, as well as personal freedom of speech in authoritarian societies. Trust metrics are used in anonymity networks to support and enhance reliability in the absence of verifiable identities, and a variety of security attacks currently focus on degrading a user's trustworthiness in the eyes of the other users. In this paper, we analyse the privacy guarantees of the CRowDs anonymity protocol, with and without onion forwarding, for standard and adaptive attacks against the trust level of honest users.
\end{abstract}

\section{Introduction}

Protecting online privacy is an essential part of today's society and its importance is increasingly recognised as crucial in many fields of computer-aided human activity, such as eVoting, eAuctions, bill payments, online betting and electronic communication. One of the most common mechanisms for privacy is anonymity, which generally refers to the condition of being unidentifiable within a given set of subjects, known as the anonymity set.

Many schemes have been proposed to enforce privacy through anonymity networks (e.g. [6, 15, 19, 24, 25]). Yet, the open nature of such networks and the unaccountability which results from the very idea of anonymity, make the existing systems prone to various attacks (e.g. [10, 18, 22, 23]). An honest user may have to suffer repeated misbehaviour (e.g., receiving infected files) without being able to identify the malicious perpetrator. Keeping users anonymous also conceals their trustworthiness, which in turn makes the information exchanged through system transactions untrustworthy as well. Consequently, a considerable amount of research has recently been focussing on the development of trust-and-reputation-based metrics aimed at enhancing the reliability of anonymity networks [7-9, 11, 31, 33.

Developing an appropriate trust metric for anonymity is very challenging, due to the fact that trust and anonymity are seemingly conflicting notions. Consider for instance the trust networks of Figure 1 In (a) peer A trusts B and D, who both trust C. Assume now that $\mathrm{C}$ wants to request a service from $\mathrm{A}$ anonymously, by proving her trustworthiness to A (i.e., the existence of a trust link to it). If $\mathrm{C}$ can prove that she is trusted by $\mathrm{D}$ without revealing her identity (using e.g. a zero-knowledge proof [3]), then A cannot distinguish whether the request originated from $\mathrm{C}$ or $\mathrm{E}$. Yet, A's trust in D could be insufficient to obtain that specific service from $A$. Therefore, $C$ could strengthen her request by proving that she is trusted by both $\mathrm{D}$ and $\mathrm{B}$. This increases the trust guarantee. Unfortunately, it also decreases C's anonymity, as A can compute the intersection of

P. Gastin and F. Laroussinie (Eds.): CONCUR 2010, LNCS 6269, pp. 48-70 2010.

(C) Springer-Verlag Berlin Heidelberg 2010 


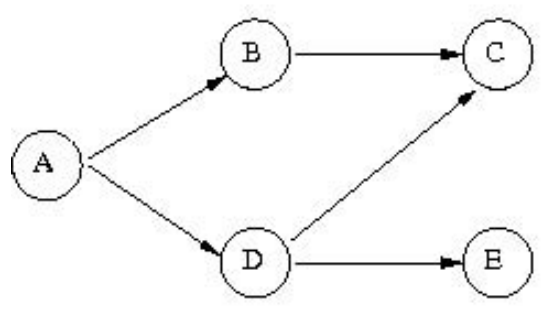

(a)

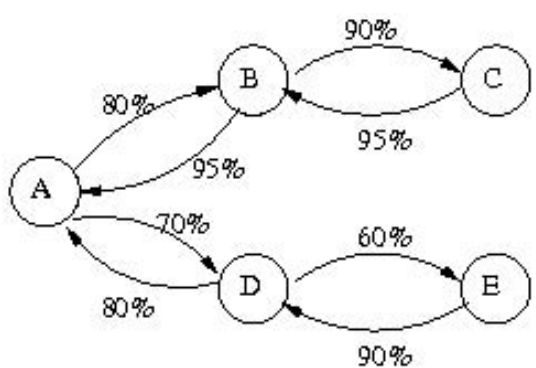

(b)

Fig. 1. Trust networks [3]

peers trusted by both $\mathrm{D}$ and $\mathrm{B}$, and therefore restrict the range of possible identities for the request's originator, or even identify $\mathrm{C}$ uniquely. Indeed, consider Figure 1 b). Here the trust level between two principals is weighted, and trust between two non-adjacent principals is computed by multiplying the values over link sequences in the obvious way. Assume that the reliability constraint is that principal $\mathrm{X}$ can send (resp. receive) a message to (from) principal $\mathrm{Y}$ if and only if her trust in $\mathrm{Y}$ is not lower than $60 \%$. Principal E can therefore only communicate through principal D. So, assuming that trust values are publicly known, E cannot possibly keep her identity from D as soon as she tries to interact at all. These examples document the existence of an inherent trade-off between anonymity and trust. The fundamental challenge is to achieve an appropriate balance between practical privacy, and acceptable network performance.

Community-based reputation systems are becoming increasingly popular both in the research literature and in practical applications. They are systems designed to estimate the trustworthiness of principals participating in some activity, as well as predict their future behaviour. Metrics for trustworthiness are primarily based on peer-review, where peers can rate each other according to the quality they experienced in their past mutual interactions [12, 13, 20]. A good reputation indicates a peer's good past behaviour, and is reflected in a high trust value. Recent research in this domain has raised fundamental issues in the design of reputation management systems for anonymous networks. In particular,

1. what metrics are suitable for computing trust for a given application field?

2. how to ensure the integrity of the peers' trust values, i.e., how to securely store and access trust values against malicious peers?

3. how to ensure that honest users accurately rate other members?

The latter issue requires a mechanism to distinguish a user's bad behaviour resulting from her being under attack, from a deliberately malicious behaviour. This is a challenging and fundamental problem. Indeed, if we cannot accurately tell these two situations apart, malicious users will target honest members in order to deteriorate their performance, and hence reduce other members' trust in them, while maintaining their apparent good behaviour. Thus, honest users may in the long term end up enjoying 
very low trust levels, while attackers might see their reputation increased, and so they increase their probability of being trusted by others. Over time this will, of course, severely affect the system's anonymity performance. Nevertheless, although a considerable effort has recently been devoted to tackle the first two issues [7, 8, 31], to the best of our knowledge the latter has been so far relatively ignored.

In this paper we investigate the effect of attacks to the trust level of honest users on the security of existing anonymity networks, such as the Reiter and Rubin's Crowds protocol [28] and onion routing networks [10].

The CRowDs protocol allows Internet users to perform anonymous web transactions by sending their messages through a random chain of users participating in the protocol. Each user in the 'crowd' must establish a path between her and a set of servers by selecting randomly some users to act as routers (or forwarders). The formation of such routing paths is performed so as to guarantee that users do not know whether their predecessors are message originators or just forwarders. Each user only has access to messages routed through her. It is well known that CRowDs cannot ensure strong anonymity in presence of corrupt participants [5, 28], yet when the number of corrupt users is sufficiently small, it provides a weaker notion of anonymity known as probable innocence. Informally, a sender is probably innocent if to an attacker she is no more likely to be the message originator than not to be.

Networks based on Onion Routing are distributed anonymising networks that use onion routing [32] to provide anonymity to their users. Similarly to Crowds, users choose randomly a path through the network in which each node knows its predecessor and successor, but no other node. The main difference with respect to Crowds is that traffic flows through the path in cells, which are created by the initiator by successively encrypting the message with the session keys of the nodes in the path, in reverse order. Each node in the act of receiving the message peels the topmost layer, discovers who the next node is, and then relays it forward. In particular, only the last node can see the message in clear and learn its final destination.

In the paper we propose two variants of the congestion attacks in the literature, aimed at deteriorating the trust level of target users in different extension of the CrowDs protocol. More specifically, we first extend the protocol so that trust is used to inform the selection of forwarding users. Our analysis of this extension shows that a DoS type attack targeting a user who initially enjoys satisfactory anonymity protection, may threaten her privacy, as her trust level quickly decreases over the time. We then extend the protocol further with a more advanced message forwarding technique, namely onion routing. While this extension offers much better protection than the previous one, our analysis ultimately shows that it suffers from similar DoS attacks as the others.

Related work. Anonymity networks date back thirty years, to when Chaum introduced the concept of Mix-net [6] for anonymous communications, where different sources send encrypted messages to a mix which forwards them to their respective destinations. Various designs [1, 10, 15, 24-26, 28, 29, 32] have since been proposed to improve Chaum's mixes, e.g., by combinations of artificial delays, variation in message ordering, encrypted message formats, message batching, and random chaining of multiple mixes.

A variety of attacks $[2,4,10,14,18,21-23,27]$ have since been discovered against such anonymity systems. Those most related to the present work are the so-called con- 
gestion or clogging attacks. In an congestion attack, the adversary monitors the flow through a node, builds paths through other nodes, and tries to use all of their available capacity [2]. The idea is that if the congested node belongs to the monitored path, the variation in the messages' arrival times will reflect at the monitored node. In [23], Murdoch and Danezis describe a congestion attack that may allow them to reveal all Tor's routers (cf. [10]) involved in a path. However, although their attack works well against a Tor network of a relatively small size, it fails against networks of typical sizes, counting nodes in the thousands. More recently, Evans et al. [14] improved Murdoch and Danezis's attack so as to practically de-anonymise Tor's users in currently deployed system. A similar attack against MorphMix [29] was recently described by Mclachlan and Hopper [21], proving wrong the previously held view that MorphMix is robust against such attacks [34]. Finally, a congestion attack is used by Hopper et al. [18] to estimate the latency between the source of a message and its first relay in Tor. In loc. cit. the authors first use a congestion attack to identify the path, and then create a parallel circuit throughout the same path to make their measurements.

Numerous denial of service (DoS) attacks have been reported in the literature. In particular, the 'packet spinning' attack of [27] tries to lure users into selecting malicious relays by targeting honest users by DoS attacks. The attacker creates long circular paths involving honest users and sends large amount of data through the paths, forcing the users to employ all their bandwidth and then timing out. These attacks motivate the demand for mechanisms to enhance the reliability of anonymity networks. In recent years, a considerable amount of research has been focusing on defining such mechanisms. In particular, trust-and-reputation-based metrics are quite popular in this domain [3, 7-9, 11, 31, 33]. Enhancing the reliability by trust, not only does improve the system's usability, but may also increase its anonymity guarantee. Indeed, a trust-based selection of relays improves both the reliability and the anonymity of the network, by delivering messages through 'trusted' routers. Moreover, the more reliable the system, the more it may attract users and hence improve the anonymity guarantee by growing the anonymity set. Introducing trust in anonymity networks does however open the flank to novel security attacks, as we prove in this paper.

In a recent paper of ours [30] we have analysed the anonymity provided by CRowDs extended with some trust information, yet against a completely different threat model. The two papers differ in several ways. Firstly, [30] considers a global and 'credentialbased' trust notion, unlike the individual-and-reputation-based trust considered here. Secondly, in [30] we considered an attack scenario where all protocol members are honest but vulnerable to being corrupted by an external attacker. The global and fixed trust in a user contrasts with the local and dynamic trust of this paper, as is meant to reflect the user's degree of resistance against corruption, that is the probability that the external attacker will fail to corrupt her. The paper derives necessary and sufficient conditions to define a 'social' policy of selecting relays nodes in order to achieve a given level of anonymity protection to all members against such attackers, as well as a 'rational' policy maximise one's own privacy.

Structure of the paper. The paper is organised as follows: in \$2 we fix some basic notations and recall the fundamental ideas of the CROwDs protocol and its properties, including the notion of probable innocence. In $\$ 3$ we present our first contribution: the 
CROwDs protocol extended with trust information in the form of a forwarding policy of its participating members, and the privacy properties of the resulting protocol are studied; $\$ 4$ repeats the analysis for an extension of the protocol with a more advanced forwarding technique inspired by onion routing. Finally, $\$ 5$ introduces a new 'adaptive' attack scenario, and presents some preliminary results on its analysis, both for the protocol with and without onion forwarding.

\section{Crowds}

In this section, we briefly revise the CRowds protocol and the notion of probable innocence.

\subsection{The Protocol}

CRowDs is a protocol proposed by Reiter and Rubin in [28] to allow Internet users to perform anonymous web transactions by protecting their identities as originators of messages. The central idea to ensure anonymity is that the originator forwards the message to another, randomly-selected user, which in turn forwards the message to a third user, and so on until the message reaches its destination (the end server). This routing process ensures that, even when a user is detected sending a message, there is a substantial probability that she is simply forwarding it on behalf of somebody else.

More specifically, a crowd consists of a fixed number of users participating in the protocol. Some members (users) of the crowd may be corrupt (the attackers), and they collaborate in order to discover the originator's identity. The purpose of the protocol is to protect the identity of the message originator from the attackers. When an originator -also known as initiator - wants to communicate with a server, she creates a random path between herself and the server through the crowd by the following process.

- Initial step: the initiator selects randomly a member of the crowd (possibly herself) and forwards the request to her. We refer to the latter user as the forwarder.

- Forwarding steps: a forwarder, upon receiving a request, flips a biased coin. With probability $1-p_{f}$ she delivers the request to the end server. With probability $p_{f}$ she selects randomly a new forwarder (possibly herself) and forwards the request to her. The new forwarder repeats the same forwarding process.

The response from the server to the originator follows the same path in the opposite direction. Users (including corrupt users) are assumed to only have access to messages routed through them, so that each user only knows the identities of her immediate predecessor and successor in the path, as well as the server.

\subsection{Probable Innocence}

Reiter and Rubin have proposed in [28] a hierarchy of anonymity notions in the context of CRowds. These range from 'absolute privacy,' where the attacker cannot perceive the presence of an actual communication, to 'provably exposed,' where the attacker can prove a sender-and-receiver relationship. Clearly, as most protocols used in practice, 
CROwDs cannot ensure absolute privacy in presence of attackers or corrupted users, but can only provide weaker notions of anonymity. In particular, in [28] the authors propose an anonymity notion called probable innocence and prove that, under some conditions on the protocol parameters, CROWDS ensures the probable innocence property to the originator. Informally, they define it as follows:

\section{A sender is probably innocent if, from the attacker's point of view, she appears no more likely to be the originator than to not be the originator.}

In other words, the attacker may have reason to suspect the sender of being more likely than any other potential sender to be the originator, but it still appears at least as likely that she is not.

We use capital letters $A, B$ to denote discrete random variables and the corresponding small letters $a, b$ and calligraphic letters $\mathcal{A}, \mathcal{B}$ for their values and set of values respectively. We denote by $P(a), P(b)$ the probabilities of $a$ and $b$ respectively and by $P(a, b)$ their joint probability. The conditional probability of $a$ given $b$ is defined as

$$
P(a \mid b)=\frac{P(a, b)}{P(b)} .
$$

Bayes Theorem relates the conditional probabilities $P(a \mid b)$ and $P(a \mid b)$ as follows

$$
P(a \mid b)=\frac{P(b \mid a) P(a)}{P(b)} .
$$

Let $n$ be the number of users participating in the protocol and let $c$ and $n-c$ be the number of the corrupt and honest members, respectively. Since anonymity makes only sense for honest users, we define the set of anonymous events as $\mathcal{A}=\left\{a_{1}, a_{2}, \ldots, a_{n-c}\right\}$, where $a_{i}$ indicates that user $i$ is the initiator of the message.

As it is usually the case in the analysis of CrowDs, we assume that attackers will always deliver a request to forward immediately to the end server, since forwarding it any further cannot help them learn anything more about the identity of the originator. Thus in any given path, there is at most one detected user: the first honest member to forward the message to a corrupt member. Therefore we define the set of observable events as $O=\left\{o_{1}, o_{2}, \ldots, o_{n-c}\right\}$, where $o_{j}$ indicates that user $j$ forwarded a message to a corrupted user. In this case we also say that user $j$ is detected by the attacker.

Reiter and Rubin [28] formalise their notion of probable innocence via the conditional probability that the initiator is detected given that any user is detected at all. This property can be written in our setting as the probability that user $i$ is detected given that she is the initiator, that is the conditional probability $P\left(o_{i} \mid a_{i}\right)$ 1 Probable innocence holds if

$$
\forall i . P\left(o_{i} \mid a_{i}\right) \leq \frac{1}{2}
$$

1 We are only interested in the case in which a user is detected, although for the sake of simplicity we shall not note that condition explicitly. 
Reiter and Rubin proved in [28] that, in CRowDs, the following holds:

$$
P\left(o_{j} \mid a_{i}\right)= \begin{cases}1-\frac{n-c-1}{n} p_{f} & i=j \\ \frac{1}{n} p_{f} & i \neq j\end{cases}
$$

Therefore, probable innocence (3) holds if and only if

$$
n \geq \frac{p_{f}}{p_{f}-1 / 2}(c+1) \quad \text { and } \quad p_{f} \geq \frac{1}{2}
$$

As previously noticed in several papers (e.g., [5]), there is a mismatch between the idea of probable innocence expressed informally by (1), and the property actually proved by Reiter and Rubin, viz. (3). The former seems indeed to correspond to the following interpretation given by Halpern and O'Neill [16]:

$$
\forall i, j . P\left(a_{i} \mid o_{j}\right) \leq \frac{1}{2} \text {. }
$$

In turn, this has been criticised for relying on the probability of users' actions, which the protocol is not really in control of, and for being too strong. However, both (3) and (5) work satisfactorily for CROwDs, thanks to its high symmetry: in fact, they coincide under its standard assumption that the a priori distribution is uniform, i.e., that each honest user has equal probability of being the initiator, which we follow in this paper too.

We remark that the concept of probable innocence was recently generalised in [17]. Instead of just comparing the probability of being innocent with the probability of being guilty, the paper focusses on the degree of innocence. Formally, given a real number $\alpha \in[0,1]$, a protocol satisfies $\alpha$-probable innocence if and only if

$$
\forall i, j . P\left(a_{i} \mid o_{j}\right) \leq \alpha
$$

Clearly $\alpha$-probable innocence coincides with standard probable innocence for $\alpha=1 / 2$.

\section{Trust in Crowds}

In the previous section, we have revised the fundamental ideas of the CRowDs protocol and its properties under the assumption that all members are deemed equal. However, as observed in $\$ 1$, this is clearly not a realistic assumption for today's open and dynamic systems. Indeed, as shown by the so-called 'packet spinning' attack [27], malicious users can attempt to make honest users select bogus routers by causing legitimate routers time out. The use attributes relating to some level of trust is therefore pivotal to enhance the reliability of the system. In this section, we firstly reformulate the CrowDS protocol under a novel scenario where the interaction between participating users is governed by their level of mutual trust; we then evaluate its privacy guarantees using property (6). We then focus on the analysis of attacks to the trust level of honest users and their impact on the anonymity of the extended protocol. Finally, we investigate the effect of a congestion attack [14] to the trust level of honest users. 


\subsection{Crowds Extended}

We now extend the CROwDs protocol to factor in a notion of trust for its participating members. To this end, we associate a trust level $t_{i j}$ to each pair of users $i$ and $j$, which represents user $i$ 's trust in user $j$. Accordingly, each user $i$ defines her policy offorwarding to other members (including herself) based on her trust in each of them. A policy of forwarding for user $i$ is a discrete probability distribution $\left\{q_{i 1}, q_{i 2}, \cdots, q_{i n}\right\}$, where $q_{i j}$ denotes the probability that $i$ chooses $j$ as the forwarder, once she has decided to forward the message.

A natural extension of CROwDs would obviously allow the initiator to select her first forwarder according to her own policy, and then leave it to the forwarder to pick the next relay, according to the forwarder's policy. This would however have the counterintuitive property that users may take part in the path which are not trusted by the initiator, just because they are trusted by a subsequent forwarder. We rather take the same view as most current systems, that the initiator is in charge of selecting the entire path which will carry her transactions. When an initiator wants to communicate with a server, she selects a random path through the crowd between herself and the server by the following process.

- First forwarder: with probability $q_{i j}$ the initiator $i$ selects a member $j$ of the crowd (possibly herself) according to her policy of forwarding $\left\{q_{i 1}, q_{i 2}, \cdots, q_{i n}\right\}$.

- Subsequent forwarders: the initiator flips a biased coin; with probability $1-p_{f}$ the current forwarder will be the last on the path, referred to as the path's exit user. Otherwise, with probability $p_{f} \times q_{i k}$, she selects $k$ (possibly herself) as the next forwarder in the path; and so on until a path's exit user is reached.

The initiator then creates the path iteratively as follows. She establishes a session key by performing an authenticated key exchange protocol, such as Diffie-Hellman 2 with the first forwarder $F_{1}$. At each of subsequent iteration $i \geq 2$, the initiator uses the partially-formed path to send $F_{i-1}$ an encrypted key exchange message to be relayed to $F_{i}$. In this way, the path is extended to $F_{i}$, and the use of session keys guarantees that any intermediary router only knows her immediate predecessor and successor. Once the path is formed, messages from the initiator to the server are sent in the same way as in the normal CRowDs. Thus, all the nodes in the path have access to the contain of the message and, obviously, to the end server. In particular, this means that the notion of detection remains the same in the extended protocol as in the original one.

Then we use our probabilistic framework to evaluate CRowds extended protocol. We start by evaluating the conditional probability $P\left(o_{j} \mid a_{i}\right)$. Let $\eta_{i}$ (resp. $\left.\zeta_{i}=1-\eta_{i}\right)$ be the overall probability that user $i$ chooses a honest (resp. corrupt) member as a forwarder. Then we have the following result.

\section{Proposition 1}

$$
\begin{array}{r}
P\left(o_{j} \mid a_{i}\right)=\zeta_{i} \epsilon_{i j}+\frac{q_{i j} \zeta_{i} p_{f}}{1-\eta_{i} p_{f}}, \\
\text { where } \eta_{i}=\sum_{k \leq(n-c)} q_{i k}, \zeta_{i}=\sum_{k \leq c} q_{i k} \text { and } \epsilon_{i j}= \begin{cases}1 & i=j \\
0 & i \neq j\end{cases}
\end{array}
$$

$\overline{2}$ We assume that public keys of participating users are known. 
Proof. Let $k$ denote the position occupied by the first honest user preceding an attacker on the path, with the initiator occupying position zero. Let $P\left(o_{j} \mid a_{i}\right)_{(k)}$ denote the probability that user $j$ is detected exactly at position $k$. Only the initiator can be detected at position zero, and the probability that this happens is equal to the overall probability that the initiator chooses a corrupt member as a forwarder. Therefore

$$
P\left(o_{j} \mid a_{i}\right)_{(0)}= \begin{cases}\zeta_{i} & i=j \\ 0 & i \neq j\end{cases}
$$

Now the probability that $j$ is detected at position $k>0$ is given by

- the probability that she decides to forward $k$ times and picks $k-1$ honest users, i.e., $p_{f}^{k-1} \eta_{i}^{k-1}$ (recall that at the initial step she does not flip the coin),

- times the probability of choosing $j$ as the $k$ th forwarder, i.e., $q_{i j}$,

- times the probability that she picks any attacker at stage $k+1$, i.e., $\zeta_{i} p_{f}$.

Therefore

$$
\forall k \geq 1, \quad P\left(o_{j} \mid a_{i}\right)_{(k)}=\eta_{i}^{k-1} p_{f}^{k} q_{i j} \zeta_{i}
$$

and hence

$$
\begin{aligned}
P\left(o_{j} \mid a_{i}\right) & =\sum_{k=0}^{\infty} P\left(o_{j} \mid a_{i}\right)_{(k)} \\
& =\zeta_{i} \epsilon_{i j}+\sum_{k=1}^{\infty} \eta_{i}^{k-1} p_{f}^{k} q_{i j} \zeta_{i} \\
& =\zeta_{i} \epsilon_{i j}+\sum_{k=0}^{\infty} \eta_{i}^{k} p_{f}^{k+1} q_{i j} \zeta_{i} \\
& =\zeta_{i} \epsilon_{i j}+p_{f} q_{i j} \zeta_{i} \sum_{k=0}^{\infty} \eta_{i}^{k} p_{f}^{k} \\
& =\zeta_{i} \epsilon_{i j}+\frac{q_{i j} \zeta_{i} p_{f}}{1-\eta_{i} p_{f}} .
\end{aligned}
$$

An immediate consequence is that when user $i$ initiates a transaction, user $j$ is not detectable if and only if the initiator's policy of forwarding never chooses an attacker or $j$ as forwarder.

Corollary 1. $P\left(o_{j} \mid a_{i}\right)=0$ if and only if one of the following holds:

1. $\zeta_{i}=0$;

2. $q_{i j}=0$ and $i \neq j$.

Now, let us compute the probability of detecting a user $P\left(o_{j}\right)$. We assume a uniform distribution for anonymous events. 
Proposition 2. If the honest members are equally likely to initiate a transaction, then

$$
P\left(o_{j}\right)=\frac{1}{n-c}\left(\zeta_{j}+\sum_{i \leq(n-c)} \frac{q_{i j} \zeta_{i} p_{f}}{1-\eta_{i} p_{f}}\right),
$$

where $\zeta_{j}$ and $\eta_{i}$ are defined as in Proposition 1

Proof. Since the anonymous events are uniformly distributed then $P\left(a_{i}\right)=1 /(n-c)$ for all $i$. Thus

$$
\begin{aligned}
P\left(o_{j}\right) & =\sum_{i \leq(n-c)} P\left(o_{j} \mid a_{i}\right) P\left(a_{i}\right) \\
& =\sum_{i \leq(n-c)} P\left(o_{j} \mid a_{i}\right) \frac{1}{n-c} \\
& =\frac{1}{n-c} \sum_{i \leq(n-c)} P\left(o_{j} \mid a_{i}\right) \\
& =\frac{1}{n-c} \sum_{i \leq(n-c)}\left(\zeta_{i} \epsilon_{i j}+\frac{q_{i j} \zeta_{i} p_{f}}{1-\eta_{i} p_{f}}\right) \\
& =\frac{1}{n-c}\left(\zeta_{j}+\sum_{i \leq(n-c)} \frac{q_{i j} \zeta_{i} p_{f}}{1-\eta_{i} p_{f}}\right) .
\end{aligned}
$$

As one could expect, a user $j$ is not detectable if both herself and any user $i$ that might include $j$ in her path never choose a corrupted member as a forwarder. Formally:

Corollary 2. $P\left(o_{j}\right)=0$ if and only if

$$
\zeta_{j}=0 \quad \text { and } \quad \forall i .\left(q_{i j}=0 \text { or } \zeta_{i}=0\right) .
$$

Now from Propositions 1 and 2 and Bayes Theorem (2), we have the following expression for the degree of anonymity provided by the extended protocol, which holds when $P\left(o_{j}\right) \neq 0$.

Proposition 3. If the honest members are equally likely to initiate a transaction, then

$$
P\left(a_{i} \mid o_{j}\right)=\frac{\zeta_{i} \epsilon_{i j}+\frac{q_{i j} \zeta_{i} p_{f}}{1-\eta_{i} p_{f}}}{\zeta_{j}+\sum_{k \leq(n-c)} \frac{q_{k j} \zeta_{k} p_{f}}{1-\eta_{k} p_{f}}},
$$

where $\zeta_{i}$ and $\eta_{j}$ are defined as above.

It is now easy to see that if all honest users have uniform probability distributions as forwarding policies, the extended protocol reduces to the original CROwDs protocol. 
Corollary 3. If for all $i$ and $j, q_{i j}=1 / n$, then $\eta_{i}=(n-c) / n$ and $\zeta_{i}=c / n$. Therefore

$$
P\left(a_{i} \mid o_{j}\right)= \begin{cases}1-\frac{n-c-1}{n} p_{f} & i=j \\ \frac{1}{n} p_{f} & i \neq j\end{cases}
$$

\subsection{On the Security of Extended CRowds}

Here we show that the absence of a uniform forwarding policy makes it very hard to achieve probable innocence as defined by Halpern and O'Neill (5). Indeed consider the following instance of the protocol, where three honest users $\{1,2,3\}$ face a single attacker $\{4\}$. Assume that the honest users are aware of the malicious behaviour of 4 , and choose their forwarding policies as follows: $p_{f}=2 / 3$, and $q_{1 j}=q_{2 j}=1 / 3$, and $q_{3 j}=0.33$ for all $j \leq 3$. In other words, the first two choose uniformly any honest users as a forwarder and never pick the attacker, whilst the third one may choose the attacker, though with a small probability $q_{34}=0.01$. Thus, $\zeta_{1}=\zeta_{2}=q_{14}=q_{24}=0$ and $\zeta_{3}=q_{34}=0.01$. It follows that $P\left(a_{3} \mid o_{j}\right)=1$, for all $j$, and the instance does not ensure probable innocence, even though the third user's policy is after all very similar to those of the other honest users. This is because if someone is detected, then user 3 is necessarily the initiator, as she is the only one who might possibly pick the attacker in her path.

Observe however that this instance of the protocol ensures probable innocence in Reiter and Rubin's formulation: indeed, for all honest users $i$ and $j, P\left(o_{j} \mid a_{i}\right)<0.0165$. The key difference at play here is that Halpern and O'Neill's definition is stronger, as it focuses on the probability that a specific user is the initiator once somebody has been detected, regardless of the probability of the detection event. On the other hand, Reiter and Rubin's formula measures exactly (the conditional probability of) the latter. This means that if the probability of detection is small, as in this case, systems may be classified as statistically secure even when one such detection event may lead to complete exposure for some initiators, as in this case.

Attackings trust. As already observed by its authors, Crowds is vulnerable to denial of service (DoS) attacks: it is enough that a single malicious router delays her forwarding action to severely hinder the viability of an entire path. This kind of attack is in fact hard for the initiator to respond to. Just because the creation of multiple paths by any single user substantially increases their security risk, the initiator has a strong incentive to keep using the degraded path. Indeed, it is advisable in CrowDs to modify a path only when it has collapsed irremediably, e.g. due to a system crash of a router, or their quitting the crowd. In this case the path is re-routed from the node preceding the failed router. As a consequence, recent research has been devoted to developing 'trust metrics' meant enhance the reliability of anonymity systems [7, 8, 31].

Although the primary goal of incorporating trust in anonymity networks is to 'enhance' the privacy guarantees by routing messages through trusted relays, preventing the presence of attackers in forwarding paths is in itself not sufficient. External attackers 


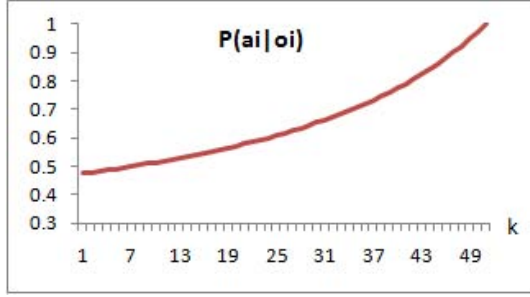

(a) $i=j=7$

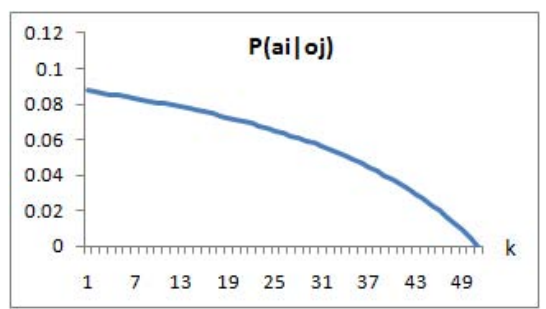

(b) $i \neq j=7$

Fig. 2. CRowds extended

may in fact target honest users with DoS attacks independent of the protocol, to make them look unreliable and/or unstable. In this way, the target users will gradually loose others members' trust, whilst internal attackers may keep accruing good reputations. Thus, over the time the trust mechanisms may become counterproductive.

Let us illustrate an attack of this kind. Consider an instance of the protocol where seven honest users $\{1,2, \cdots, 7\}$ face a single attacker $\{8\}$, assume that 7 is the honest user targeted by the attack, and that all users are equally likely to initiate a transaction. Recall that a path in CROwDs remains fixed for a certain amount of time -typically one day- known as a session. In practice, all transactions initiated by a given user follow the same path, regardless of their destination servers. At the end of the session then, all existing paths are destroyed, new members can join the crowd, and each member willing to initiate anonymous transactions creates a new path. Trust level updates play therefore their role at the beginning of each session. For the purpose of this example, we assume that the protocol is equipped with mechanisms to detect unstable routers (e.g., by monitoring loss of messages, timeouts, variations in response time and so on); upon realising that her path is unstable, an initiator will notify all members of the identity of the unstable node (in this case 7) 3 When a node is reported as unstable, all other honest nodes decrease their trust in her at the beginning of the following session. For simplicity, we assume that all users start with the same trust level $\tau$, and that the target user remains fixed over time. The following policies of forwarding are therefore in place for each session, with $n=8, c=1$ and $\tau=50$.

$$
q_{i j}^{(k)}=\left\{\begin{array}{cl}
\frac{1}{n} & i=7 \\
\frac{\tau-k}{n \times \tau-k} & i \neq 7 \text { and } j=7 \\
\frac{\tau}{n \times \tau-k} & i \neq 7 \text { and } j \neq 7 .
\end{array}\right.
$$

In words, honest users other that the target decrease their trust in her by one and redistributed it uniformly to the remaining users. On the other hand, the target has no

\footnotetext{
3 This contrasts with the approach of [11], where the initiator would directly decrease her trust in all users in the path.
} 
reason to change her trust, as there is no evidence to suspect anybody as the source of the external attack. Thus, her policy remains the same over the time. Hence, we have

$$
\zeta_{i}^{(k)}=\left\{\begin{array}{cl}
\frac{c}{n} & i=7 \\
\frac{\tau}{n \times \tau-k} & \text { otherwise. }
\end{array}\right.
$$

Assuming that the forwarding probability is $p_{f}=0.7$, Figure 2 shows the probability that the target will be identified over time. Clearly, the target's privacy deteriorates quickly, as it becomes increasingly unlikely that users other than herself pick her when building a path. In particular, after seven sessions the protocol can no longer ensure probable innocence as the probability $P\left(a_{7} \mid o_{7}\right)$ becomes greater than 0.5 .

\section{Onion Forwarding in Crowds}

In the previous section we analysed the privacy protection afforded by CRowDs extended with a notion of trust. Following a similar pattern, in this section we focus on the privacy guarantees offered by our protocol when equipped with 'onion forwarding,' a superior forwarding technique used in systems actually deployed, such as Tor [10].

In CROwDs, any user participating in a path has access to the cleartext messages routed through it. In particular, as all relay requests expose the message's final destination, a team of attackers will soon build up a host of observations suitable to classify the behaviour of honest participants. We recently proved in [17] that such extra attackers' knowledge makes it very difficult to achieve anonymity in CRowDs. The most effective technique available against such a risk is onion forwarding, originally used in the 'Onion Routing' protocol [32], and currently implemented widely in real-world systems. The idea is roughly as follows. When forming a path, the initiator establishes a set of session encryption keys, one for each user in it, which she then uses to repeatedly encrypt each message she routes through, starting with the last node on the path, and ending with the first. Each intermediate user, in the act of receiving the message decrypts it with her key. Doing so, she 'peels' away the outmost layer of encryption, discovers who the next forwarder is, and relays the message as required. In particular, only the last node sees the message in clear and learns its actual destination. Thus, a transaction is detected only if the last user in the path, also known as the 'exit node,' is an attacker, and the last honest user in the path is then detected.

\subsection{Privacy Level of the Onion Forwarding}

Next we study the privacy ensured to each member participating in the protocol under the onion forwarding scheme. As we did earlier, we begin with computing the conditional probability $P\left(o_{j} \mid a_{i}\right)$.

\section{Proposition 4}

$$
P\left(o_{j} \mid a_{i}\right)=\left(1-p_{f}\right) \zeta_{i} \epsilon_{i j}+\frac{q_{i j} \zeta_{i} p_{f}}{1-\zeta_{i} p_{f}} .
$$


Proof. Let $k$ denote the last position occupied by an honest user preceding an attacker on the path, i.e., the position of the detected user. We denote by $P\left(o_{j} \mid a_{i}\right)_{(k)}$ the probability that user $j$ is detected exactly at position $k$. Again, only the initiator can be detected at position zero, and the probability that this happens is equal to the overall probability that the initiator chooses a corrupt member as a forwarder, multiplied by the probability that the latter is the last node in the path. Therefore

$$
P\left(o_{j} \mid a_{i}\right)_{(0)}= \begin{cases}\left(1-p_{f}\right) \zeta_{i} & i=j \\ 0 & i \neq j\end{cases}
$$

Now the probability that $j$ is detected at position $k>0$ is given by

- the probability that she decides to forward $k$ times and picks $k-1$ users (does not matter whether honest or not, as non-exit attackers cannot see the messages), i.e., $p_{f}^{k-1}$ (recall that at the initial step she does not flip the coin),

- times the probability of choosing $j$ as the $k$ th forwarder, i.e. $q_{i j}$,

- times the probability that she picks any number $k^{\prime}$ of attackers at the end of the path, i.e. $\sum_{k^{\prime}=1}^{\infty} p_{f}^{k^{\prime}} \zeta_{i}^{k^{\prime}}\left(1-p_{f}\right)$.

Therefore

$$
\forall k \geq 1, \quad P\left(o_{j} \mid a_{i}\right)_{(k)}=\sum_{k=1}^{\infty}\left(p_{f}^{k-1} q_{i j} \sum_{k^{\prime}=1}^{\infty} p_{f}^{k^{\prime}} \zeta_{i}^{k^{\prime}}\left(1-p_{f}\right)\right),
$$

and hence

$$
\begin{aligned}
P\left(o_{j} \mid a_{i}\right) & =\sum_{k=0}^{\infty} P\left(o_{j} \mid a_{i}\right)_{(k)} \\
& =\left(1-p_{f}\right) \zeta_{i} \epsilon_{i j}+\sum_{k=1}^{\infty}\left(p_{f}^{k-1} q_{i j} \sum_{k^{\prime}=1}^{\infty} p_{f}^{k^{\prime}} \zeta_{i}^{k^{\prime}}\left(1-p_{f}\right)\right) \\
& =\left(1-p_{f}\right)\left[\zeta_{i} \epsilon_{i j}+q_{i j} \sum_{k=1}^{\infty}\left(p_{f}^{k-1} \sum_{k^{\prime}=1}^{\infty} p_{f}^{k^{\prime}} \zeta_{i}^{k^{\prime}}\right)\right] \\
& =\left(1-p_{f}\right)\left[\zeta_{i} \epsilon_{i j}+q_{i j} \sum_{k=1}^{\infty} p_{f}^{k-1} \frac{\zeta_{i} p_{f}}{1-\zeta_{i} p_{f}}\right] \\
& =\left(1-p_{f}\right)\left[\zeta_{i} \epsilon_{i j}+\frac{q_{i j} \zeta_{i} p_{f}}{1-\zeta_{i} p_{f}} \frac{1}{1-p_{k}}\right] \\
& =\left(1-p_{f}\right)\left[\zeta_{i} \epsilon_{i j}+\frac{q_{i j} \zeta_{i} p_{f}}{\left(1-p_{f}\right)\left(1-\zeta_{i} p_{f}\right)}\right]
\end{aligned}
$$

Corollary 4. $P\left(o_{j} \mid a_{i}\right)=0$ if and only if one of the following holds:

1. $\zeta_{i}=0$;

2. $q_{i j}=0$ and $i \neq j$. 
Now on the probability of detecting a user $P\left(o_{j}\right)$. Assuming uniform distribution of anonymous events we have the following result.

Proposition 5. If the honest member are equally likely to initiate a transaction then.

$$
P\left(o_{j}\right)=\frac{1}{n-c}\left(\left(1-p_{f}\right) \zeta_{j}+\sum_{i \leq(n-c)} \frac{q_{i j} \zeta_{i} p_{f}}{1-\zeta_{i} p_{f}}\right) .
$$

Proof. Since the anonymous events are uniformly distributed then $P\left(a_{i}\right)=1 /(n-c)$ for all $i$. Thus

$$
\begin{aligned}
P\left(o_{j}\right) & =\sum_{i \leq(n-c)} P\left(o_{j} \mid a_{i}\right) P\left(a_{i}\right) \\
& =\sum_{i \leq(n-c)} P\left(o_{j} \mid a_{i}\right) \frac{1}{n-c} \\
& =\frac{1}{n-c} \sum_{i \leq(n-c)} P\left(o_{j} \mid a_{i}\right) \\
& =\frac{1}{n-c} \sum_{i \leq(n-c)}\left(\left(1-p_{f}\right) \zeta_{i} \epsilon_{i j}+\frac{q_{i j} \zeta_{i} p_{f}}{1-\zeta_{i} p_{f}}\right) \\
& =\frac{1}{n-c}\left(\left(1-p_{f}\right) \zeta_{j}+\sum_{i \leq(n-c)} \frac{q_{i j} \zeta_{i} p_{f}}{1-\zeta_{i} p_{f}}\right) .
\end{aligned}
$$

We then have the same conditions of non-detectability as in the previous section; that is, the following result holds.

Corollary 5. $P\left(o_{j}\right)=0$ if and only if

$$
\zeta_{j}=0 \quad \text { and } \forall i .\left(q_{i j}=0 \text { or } \zeta_{i}=0\right) .
$$

Now from Proposition 4 and 5 and the Bayes theorem, we have the following result.

Proposition 6. If the honest members are equally likely to initiate a transaction, then

$$
P\left(a_{i} \mid o_{j}\right)=\frac{\zeta_{i} \epsilon_{i j}+\frac{q_{i j} \zeta_{i} p_{f}}{\left(1-p_{f}\right)\left(1-\zeta_{i} p_{f}\right)}}{\zeta_{j}+\sum_{k \leq(n-c)} \frac{q_{k j} \zeta_{k} p_{f}}{\left(1-p_{f}\right)\left(1-\zeta_{k} p_{f}\right)}} .
$$

Now from Propositions 3 and 6 , we can prove effectively that the privacy level ensured by the onion version is better than those offered by the versions where messages are forwarded in cleartext. More formally, let $\left[P\left(a_{i} \mid o_{j}\right)\right]_{C R}$ and $\left[P\left(a_{i} \mid o_{j}\right)\right]_{O R}$ denote the probability that $i$ is the initiator given that $j$ is detected under cleartext routing and onion routing, respectively. Then the following holds.

Theorem 1. $\left[P\left(a_{i} \mid o_{j}\right)\right]_{O R} \leq\left[P\left(a_{i} \mid o_{j}\right)\right]_{C R}$. 


\subsection{On the Security of the Onion Forwarding Version}

As mentioned before, onion forwarding is the forwarding technique of choice in several real-world systems. Recent work [14, 18, 21-23] shows that such systems are vulnerable to so-called congestion attacks, which intuitively work as follows. Assume that the initiator selects a path which contains a corrupt user as the exit node. The attacker can then observe the pattern of arrival times of the initiator's requests, and tries to identify the entire path by selectively congesting the nodes she suspect to belong to it. Precisely, to determine whether or not a specific node occurs in the path, she asks a collaborating attacker to build a long path looping on the target node and ending with a corrupt node. Using this, the attacker perturbs the flow through the target node, so that if the latter belongs also to the path under observation, the perturbation will reflect at its exit node.

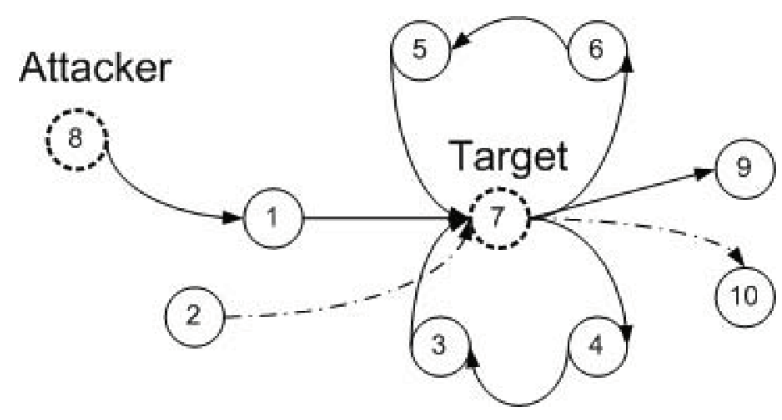

Fig. 3. Congestion attack

Here we use a variant of the congestion attack which, similarly to the previous section, allows internal attackers to deteriorate the reputation of a targeted honest user, and does not require the attacker to belong to a path. Figure 3 illustrates the attack, where a long path is built looping as many times as possible over the target, preferably using different loops involving different users. Thank to such properties, the target user will be significantly busy handling the same message again and again, whilst no other member of the path will be congested.

Figure 4illustrates the effect of this attack using the same example as in the cleartext forwarding version in $\$ 3$. The results are completely in tune with those presented by Figure 2, even though the target node initially enjoys a better anonymity protection, her anonymity will unequivocally fall, although more smoothly than in $\$ 3$ In particular, after twenty sessions, the protocol no longer ensures probable innocence, as the probability of identifying the target node becomes greater than 0.5 .

\section{Adaptive Attackers}

We have worked so far under the assumption that protocol participants either behave always honestly or always maliciously. Arguably, this is a rather unrealistic hypothesis 


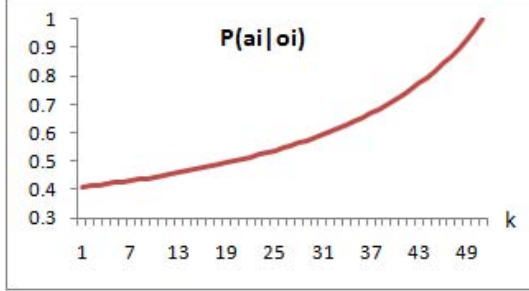

(a) $i=j=7$

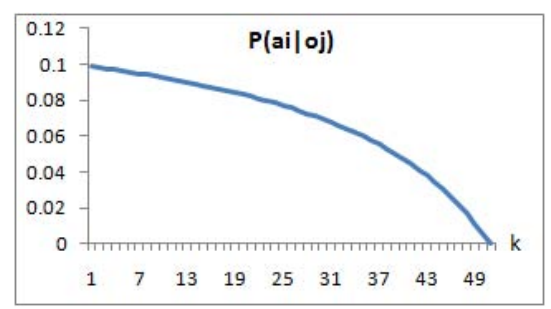

(b) $i \neq j=7$

Fig. 4. Onion forwarding

in open and dynamic systems, where honest nodes can become malicious upon being successfully attacked. In this section we take the more realistic view that nodes may become corrupt, and study a new kind of attackers, which we dub 'adaptive,' and the relative attacks.

Adaptive attackers differ from those we considered so far in the paper-and indeed from those considered so far in the literature on CRowDs- in that when they intercept a message, rather than just reporting its sender as the initiator, they attempt to travel the path back in order to improve their chance to catch the actual originator. They do so by trying to corrupt the sender of the message, say $j_{1}$. If the attack succeeds, then the attacker effectively learns from $j_{1}$ all she needs to identify $j_{1}$ 's predecessor on the path, say $j_{2}$, and repeat the adaptive attack on $j_{2}$, having moved a step closer to the initiator. The process is repeated iteratively until the attacker either fails to corrupt the current node (or timeouts whilst trying to) or reaches the beginning of the path. When that happens, the attacker reports the current node, say $j_{k}$, which is obviously a better candidate than $j_{1}$ to have originated the transaction.

We regard this as a significant and realistic kind of attack, as there clearly are a multitude of ways in which the adaptive attacker may attempt to corrupt a node. These range from brute force attacks via virus and worms which gains the attacker complete control over the node, to milder approaches based on luring the target to give away some bit of information in exchange for some form of benefit, and in general are entirely independent of the CRowds protocol. We therefore do not postulate here about the means which may be available to the attacker to carry out her task, make no assumptions whatsoever about her power, and take the simplified view that each node has at all time the same probability $\pi$ to become corrupted.

In the rest of the section we re-evaluate the privacy guarantees afforded by CrowDs extended -with and without onion forwarding- under this new adaptive attack scenario. We shall however carry out the analysis under the unrealistic assumption that it is necessary for attackers to corrupt a node each time they meet her on the path. Recall in fact that a single node will typically appear several times in a path. Therefore, an adaptive attacker in her attempt to travel the path backwards towards the initiator will in general meet the each node several times. The reason why our assumption may be justified is when the attacks only gain the attacker access to just enough data to get to the node's last predecessor on the path, rather than to the entire set of them. On the other hand, the 
reason why this assumption is ultimately unsatisfactory is that it is overly dangerous to make limiting assumptions as to the degree of success of an attack, and assess speculatively the extent to which a node's integrity is compromised, the methodologically correct attitude being to assume that the attacker has gained total control over the target. And when she has, by definition she simply has no need to corrupt the node again, and no new knowledge may be acquired by doing so. In the concluding section, we discuss a few preliminary ideas on how to remove this restriction in future work.

\subsection{Crowds Extended}

Our technical development proceeds mutatis mutandis as in $\$ 3$ and $\$ 4$ In particular, as before we first evaluate the conditional probability $P\left(o_{j} \mid a_{i}\right)$, then under the hypothesis that all honest users are equally likely to initiate a transaction, we compute $P\left(o_{j}\right)$, and finally, using Bayes Theorem, we obtain $P\left(a_{i} \mid o_{j}\right)$. In this section we omit all proofs.

The probabilities $P\left(o_{i} \mid a_{i}\right)_{(0)}$ and $P\left(o_{j} \mid a_{i}\right)_{(1+)}$ that node $i$ is detected at the initiator position or at any position after that can be expressed respectively as

$$
\begin{gathered}
P\left(o_{i} \mid a_{i}\right)_{(0)}=\zeta_{i}+\frac{p_{f} \eta_{i} \zeta_{i} \pi}{1-\pi}\left(\frac{1}{1-p_{f} \eta_{i}}-\frac{\pi}{1-\pi p_{f} \eta_{i}}\right), \\
P\left(o_{j} \mid a_{i}\right)_{(1+)}=\frac{q_{i j} \zeta_{i} p_{f}}{1-p_{f} \eta_{i}}-\frac{q_{i j} \zeta_{i} \eta_{i} p_{f}^{2} \pi^{2}}{(1-\pi)\left(1-p_{f} \zeta_{i}\right)},
\end{gathered}
$$

which gives the following result, where again $\epsilon_{i j}=1$ if $i=j$, and 0 otherwise.

The key to these formulae is to consider that when a user is detected at position $h$, this is potentially due to a detection at position $h+k$, for any $k \geq 0$, whereby the attacker has successively travelled back $k$ positions on the path, by either corrupting honest users with probability $\pi$ or by meeting other attackers. The situation would be quite different were we to take into account that the attacker only needs to corrupt a honest user once, as $\pi$ would not anymore be a constant.

\section{Proposition 7}

$$
P\left(o_{j} \mid a_{i}\right)=\epsilon_{i j} P\left(o_{i} \mid a_{i}\right)_{(0)}+P\left(o_{j} \mid a_{i}\right)_{(1+)} .
$$

Under the hypothesis of a uniform distribution of anonymous events, it is easy to prove the following.

Proposition 8. If the honest members are equally likely to initiate a transaction, then

$$
P\left(o_{j}\right)=\frac{1}{n-c}\left(P\left(o_{j} \mid a_{j}\right)_{(0)}+\sum_{k \leq(n-c)} P\left(o_{j} \mid a_{k}\right)_{(1+)}\right) .
$$

Now from Proposition 7 and 8 and Bayes Theorem, we have the following. 


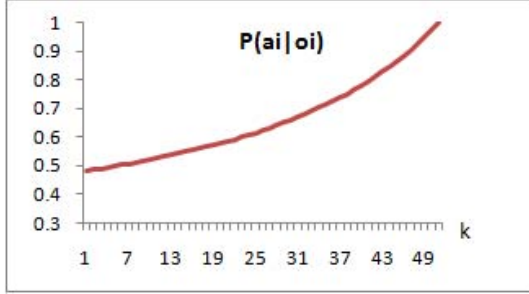

(a) $i=j=7, \pi=0.02$

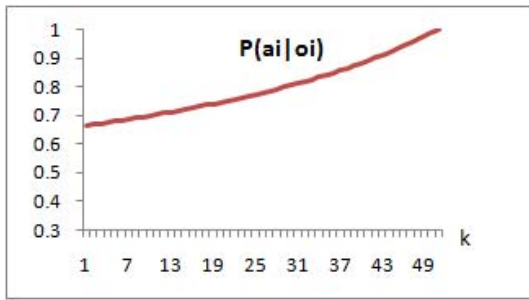

(c) $i=j=7, \pi=0.5$

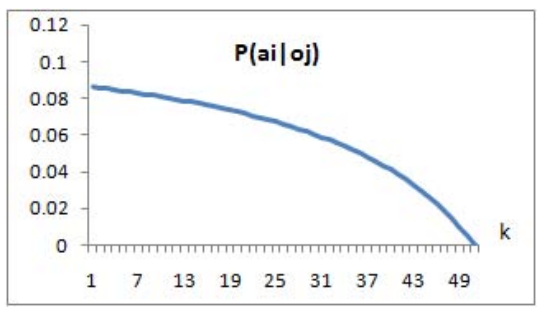

(b) $i \neq j=7, \pi=0.02$

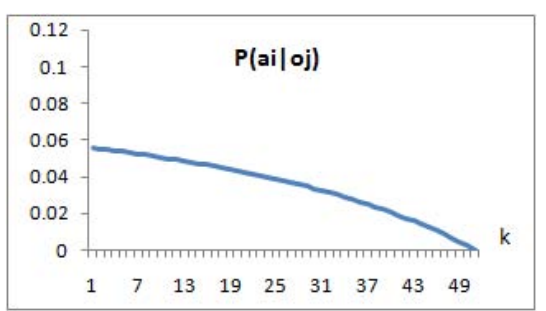

(d) $i \neq j=7, \pi=0.5$

Fig. 5. Example in Crowds extended against adaptive attack

Proposition 9. If the honest members are equally likely to initiate a transaction, then

$$
P\left(a_{i} \mid o_{j}\right)=\frac{\epsilon_{i j} P\left(o_{i} \mid a_{i}\right)_{(0)}+P\left(o_{j} \mid a_{i}\right)_{(1+)}}{P\left(o_{j} \mid a_{j}\right)_{(0)}+\sum_{k \leq(n-c)} P\left(o_{j} \mid a_{k}\right)_{(1+)}} .
$$

Of course, in case the attacker's attempts to travel back the path never succeed, the formula reduces to the one we found previously.

Corollary 6. If $\pi=0$, that is the attacker is not adaptive, then

$$
P\left(a_{i} \mid o_{j}\right)=\frac{\zeta_{i} \epsilon_{i j}+\frac{q_{i j} \zeta_{i} p_{f}}{1-\eta_{i} p_{f}}}{\zeta_{j}+\sum_{k \leq(n-c)} \frac{q_{k j} \zeta_{k} p_{f}}{1-\eta_{k} p_{f}}},
$$

which is the same as Proposition 3

Figure 5 illustrates the formulae $P\left(a_{7} \mid o_{7}\right)$ and $P\left(a_{i} \mid o_{7}\right)$ for $i \neq 7$ on our running example, where we add $\pi=0.02$ and $\pi=0.5$ to the existing parameters, viz., $n=8, c=1$, $p_{f}=0.7$, and $\tau=50$. It is interesting here to observe the effect of the attacker's corruption power, insofar as that is represented by $\pi$ : the larger $\pi$, the more lethal the attacker, the farther away the protocol from the standard, and the more insecure. In particular, for $\pi=0.5$ the system fails by a large margin to guarantee probable innocence even before the attack to 7's trust level starts. 


\subsection{Onion Forwarding}

Under onion forwarding, the adaptive attackers must appear as the last node on the path, and from there, starting with her predecessor, try to corrupt nodes back towards the originator. Following the same proof strategy as before, we define obtain the following formulae.

$$
\begin{aligned}
& P\left(o_{i} \mid a_{i}\right)_{(0)}=\left(1-p_{f}\right) \zeta_{i}+\frac{p_{f} \eta_{i} \zeta_{i} \pi\left(1-p_{f}\right)}{\left(1-p_{f} \zeta_{i}\right)(1-\pi)}\left(\frac{1}{1-\eta_{i} p_{f}}-\frac{\pi}{1-\pi \eta_{i} p_{f}}\right), \\
& P\left(o_{j} \mid a_{i}\right)_{(1+)}=\frac{q_{i j} \zeta_{i} p_{f}}{1-\zeta_{i} p_{f}}+\frac{p_{f}^{2} \eta_{i} \zeta_{i} \pi q_{i j}}{\left(1-p_{f} \zeta_{i}\right)(1-\pi)}\left(\frac{1}{1-\eta_{i} p_{f}}-\frac{\pi}{1-\pi \eta_{i} p_{f}}\right),
\end{aligned}
$$

and therefore:

\section{Proposition 10}

$$
P\left(o_{j} \mid a_{i}\right)=\epsilon_{i j} P\left(o_{i} \mid a_{i}\right)_{(0)}+P\left(o_{j} \mid a_{i}\right)_{(1+)} .
$$

Now on the probability of detecting a user $P\left(o_{j}\right)$.

Proposition 11. If the honest members are equally likely to initiate a transaction, then

$$
P\left(o_{j}\right)=\frac{1}{n-c}\left(P\left(o_{j} \mid a_{j}\right)_{(0)}+\sum_{k \leq(n-c)} P\left(o_{j} \mid a_{k}\right)_{(1+)}\right) .
$$

As before, the result below follows from Propositions 10 and 11 and Bayes Theorem.

Proposition 12. If the honest members are equally likely to initiate a transaction then.

$$
P\left(a_{i} \mid o_{j}\right)=\frac{\epsilon_{i j} P\left(o_{i} \mid a_{i}\right)_{(0)}+P\left(o_{j} \mid a_{i}\right)_{(1+)}}{P\left(o_{j} \mid a_{j}\right)_{(0)}+\sum_{k \leq(n-c)} P\left(o_{j} \mid a_{k}\right)_{(1+)}} .
$$

Corollary 7. If $\pi=0$, that is the attacker after all not adaptive, then

$$
P\left(a_{i} \mid o_{j}\right)=\frac{\zeta_{i} \epsilon_{i j}+\frac{q_{i j} \zeta_{i} p_{f}}{\left(1-p_{f}\right)\left(1-\zeta_{i} p_{f}\right)}}{\zeta_{j}+\sum_{k \leq(n-c)} \frac{q_{k j} \zeta_{k} p_{f}}{\left(1-p_{f}\right)\left(1-\zeta_{k} p_{f}\right)}},
$$

which coincides with Proposition 6.

Finally, Figure 6 illustrates $P\left(a_{7} \mid o_{7}\right)$ and $P\left(a_{i} \mid o_{7}\right)$ for $i \neq 7$ on our running example, for $\pi=0.5$. Although the graphs are shaped as in the previous cases, it is possible to notice the increase security afforded by the onion forwarding. 


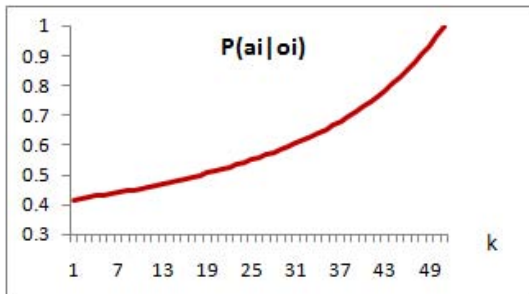

(a) $i=j=7$

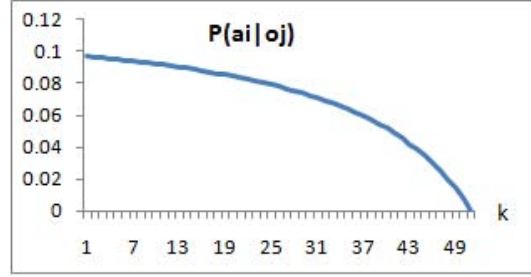

(b) $i \neq j=7$

Fig. 6. Onion forwarding against adaptive attacks

\section{Conclusion}

In this paper we have presented an enhancement of the CRowDs anonymity protocol via a notion of trust which allows crowd members to route their traffic according to their perceived degree of trustworthiness of other members. We formalised the idea quite simply by means of (variable) forwarding policies, with and without onion forwarding techniques. Our protocol variation has the potential of improving the overall trustworthiness of data exchanges in anonymity networks, which may naturally not be taken for granted in a context where users are actively trying to conceal their identities. We then analysed the privacy properties of the protocol quantitatively, both for CROWDs and onion forwarding, under standard and adaptive attacks.

Our analysis in the case of adaptive attacks is incomplete, in that it assumes that attackers whilst attempting to travel back over a path towards its originator, need to corrupt each honest node each time they meet her. Arguably, this is not so. Typically a node $j$ will act according to a routing table, say $T_{j}$. This will contain for each path's id a translation id and a forwarding address (either another user, or the destination server) and, in the case of onion forwarding, the relevant encryption key. (Observe that since path's id are translated at each step, $j$ may not be able to tell whether or not two entries in $T_{j}$ actually correspond to a same path and, therefore, may not know how many times she occurs on each path.) It is reasonable to assume that upon corruption an attacker $c$ will seize $T_{j}$, so that if she ever reaches $j$ again, $c$ will find all the information to continue the attack just by inspecting $T_{j}$.

Observe now that the exact sequence of users in the path is largely irrelevant to compute $P\left(o_{j} \mid a_{i}\right)$. It only matters how many times each of them appears in between the attacker at the end of the path and the detected node. Using some combinatorics, it is therefore relatively easy to write a series for $P\left(o_{j} \mid a_{i}\right)$ based on summing up a weighted probability for all possible occurrence patterns of $n-c$ honest users and $c$ attackers in the path. Quite a different story is to simplify that series to distill a usable formula. That is a significant task which we leave for future work.

Acknowledgements. We thank Ehab ElSalamouny and Catuscia Palamidessi for their insights and for proofreading. 


\section{References}

1. Abe, M.: Universally verifiable Mix-net with verification work indendent of the number of Mix-servers. In: Nyberg, K. (ed.) EUROCRYPT 1998. LNCS, vol. 1403, pp. 437-447. Springer, Heidelberg (1998)

2. Back, A., Möller, U., Stiglic, A.: Traffic analysis attacks and trade-offs in anonymity providing systems. In: Moskowitz, I.S. (ed.) IH 2001. LNCS, vol. 2137, pp. 245-257. Springer, Heidelberg (2001)

3. Backes, M., Lorenz, S., Maffei, M., Pecina, K.: Anonymous webs of trust. In: 10th Privacy Enhancing Technologies Symposium, PETS 2010. LNCS. Springer, Heidelberg (to appear, 2010)

4. Borisov, N., Danezis, G., Mittal, P., Tabriz, P.: Denial of service or denial of security? In: Ning, P., di Vimercati, S.D.C., Syverson, P.F. (eds.) ACM Conference on Computer and Communications Security, pp. 92-102. ACM, New York (2007)

5. Chatzikokolakis, K., Palamidessi, C.: Probable innocence revisited. Theor. Comput. Sci. 367(1-2), 123-138 (2006)

6. Chaum, D.: Untraceable electronic mail, return addresses, and digital pseudonyms. Commun. ACM 24(2), 84-88 (1981)

7. Damiani, E., di Vimercati, S.D.C., Paraboschi, S., Pesenti, M., Samarati, P., Zara, S.: Fuzzy logic techniques for reputation management in anonymous peer-to-peer systems. In: Wagenknecht, M., Hampel, R. (eds.) Proceedings of the 3rd Conference of the European Society for Fuzzy Logic and Technology, pp. 43-48 (2003)

8. Damiani, E., di Vimercati, S.D.C., Paraboschi, S., Samarati, P., Violante, F.: A reputationbased approach for choosing reliable resources in peer-to-peer networks. In: Atluri, V. (ed.) ACM Conference on Computer and Communications Security, pp. 207-216. ACM, New York (2002)

9. Dingledine, R., Freedman, M.J., Hopwood, D., Molnar, D.: A reputation system to increase mix-net reliability. In: Moskowitz, I.S. (ed.) IH 2001. LNCS, vol. 2137, pp. 126-141. Springer, Heidelberg (2001)

10. Dingledine, R., Mathewson, N., Syverson, P.F.: Tor: The second-generation onion router. In: USENIX Security Symposium, pp. 303-320. USENIX (2004)

11. Dingledine, R., Syverson, P.F.: Reliable MIX cascade networks through reputation. In: Blaze, M. (ed.) FC 2002. LNCS, vol. 2357, pp. 253-268. Springer, Heidelberg (2003)

12. ElSalamouny, E., Krukow, K.T., Sassone, V.: An analysis of the exponential decay principle in probabilistic trust models. Theor. Comput. Sci. 410(41), 4067-4084 (2009)

13. ElSalamouny, E., Sassone, V., Nielsen, M.: HMM-based trust model. In: Degano, P., Guttman, J.D. (eds.) Formal Aspects in Security and Trust. LNCS, vol. 5983, pp. 21-35. Springer, Heidelberg (2010)

14. Evans, N.S., Dingledine, R., Grothoff, C.: A practical congestion attack on Tor using long paths. In: Proceedings of the 18th USENIX Security Symposium (2009)

15. Freedman, M.J., Morris, R.: Tarzan: a peer-to-peer anonymizing network layer. In: Atluri, V. (ed.) ACM Conference on Computer and Communications Security, pp. 193-206. ACM, New York (2002)

16. Halpern, J.Y., O'Neill, K.R.: Anonymity and information hiding in multiagent systems. Journal of Computer Security 13(3), 483-512 (2005)

17. Hamadou, S., Palamidessi, C., Sassone, V., ElSalamouny, E.: Probable innocence in the presence of independent knowledge. In: Degano, P., Guttman, J.D. (eds.) Formal Aspects in Security and Trust. LNCS, vol. 5983, pp. 141-156. Springer, Heidelberg (2010)

18. Hopper, N., Vasserman, E.Y., Chan-Tin, E.: How much anonymity does network latency leak? ACM Trans. Inf. Syst. Secur. 13(2) (2010) 
19. Jakobsson, M.: Flash mixing. In: Annual ACM Symposium on Principles of Distributed Computing, PODC 1999, pp. 83-89 (1999)

20. Krukow, K., Nielsen, M., Sassone, V.: A logical framework for history-based access control and reputation systems. Journal of Computer Security 16(1), 63-101 (2008)

21. McLachlan, J., Hopper, N.: Don't clog the queue! circuit clogging and mitigation in P2P anonymity schemes. In: Tsudik, G. (ed.) FC 2008. LNCS, vol. 5143, pp. 31-46. Springer, Heidelberg (2008)

22. McLachlan, J., Tran, A., Hopper, N., Kim, Y.: Scalable onion routing with Torsk. In: AlShaer, E., Jha, S., Keromytis, A.D. (eds.) ACM Conference on Computer and Communications Security, pp. 590-599. ACM, New York (2009)

23. Murdoch, S.J., Danezis, G.: Low-cost traffic analysis of tor. In: IEEE Symposium on Security and Privacy, pp. 183-195. IEEE Computer Society, Los Alamitos (2005)

24. Nambiar, A., Wright, M.: Salsa: a structured approach to large-scale anonymity. In: Juels, A., Wright, R.N., di Vimercati, S.D.C. (eds.) ACM Conference on Computer and Communications Security, pp. 17-26. ACM, New York (2006)

25. Neff, C.A.: A verifiable secret shuffle and its application to e-voting. In: ACM Conference on Computer and Communications Security, pp. 116-125 (2001)

26. Ohkubo, M., Abe, M.: A length-invariant hybrid mix. In: Okamoto, T. (ed.) ASIACRYPT 2000. LNCS, vol. 1976, pp. 178-191. Springer, Heidelberg (2000)

27. Pappas, V., Athanasopoulos, E., Ioannidis, S., Markatos, E.P.: Compromising anonymity using packet spinning. In: Wu, T.-C., Lei, C.-L., Rijmen, V., Lee, D.-T. (eds.) ISC 2008. LNCS, vol. 5222, pp. 161-174. Springer, Heidelberg (2008)

28. Reiter, M.K., Rubin, A.D.: Crowds: Anonymity for web transactions. ACM Trans. Inf. Syst. Secur. 1(1), 66-92 (1998)

29. Rennhard, M., Plattner, B.: Introducing MorphMix: peer-to-peer based anonymous internet usage with collusion detection. In: Jajodia, S., Samarati, P. (eds.) Proceedings of the 2002 ACM workshop on Privacy in the Electronic Society, WPES, pp. 91-102. ACM, New York (2002)

30. Sassone, V., ElSalamouny, E., Hamadou, S.: Trust in Crowds: probabilistic behaviour in anonymity protocols. In: Symposium on Trustworthy Global Computing, TGC 2010. LNCS, vol. 6084. Springer, Heidelberg (2010)

31. Singh, A., Liu, L.: Trustme: Anonymous management of trust relationships in decentralized P2P systems. In: Shahmehri, N., Graham, R.L., Caronni, G. (eds.) Peer-to-Peer Computing, pp. 142-149. IEEE Computer Society, Los Alamitos (2003)

32. Syverson, P.F., Goldschlag, D.M., Reed, M.G.: Anonymous connections and onion routing. In: IEEE Symposium on Security and Privacy, pp. 44-54. IEEE Computer Society, Los Alamitos (1997)

33. Wang, Y., Vassileva, J.: Trust and reputation model in peer-to-peer networks. In: Shahmehri, N., Graham, R.L., Caronni, G. (eds.) Peer-to-Peer Computing. IEEE Computer Society, Los Alamitos (2003)

34. Wiangsripanawan, R., Susilo, W., Safavi-Naini, R.: Design principles for low latency anonymous network systems secure against timing attacks. In: Brankovic, L., Coddington, P.D., Roddick, J.F., Steketee, C., Warren, J.R., Wendelborn, A.L. (eds.) Proc. Fifth Australasian Information Security Workshop (Privacy Enhancing Technologies), AISW 2007. CRPIT, vol. 68, pp. 183-191. Australian Computer Society (2007) 